Estudios Románicos, Volumen 29, 2020, pp. 73-78

ISSN: 0210-4911

eISSN: $1989-614 \mathrm{X}$

DOI: https://doi.org/10.6018/ER.427031

\title{
LA EMOCIÓN DEL PAISAJE EN EL JOVEN AZORÍN
}

(The emotion of the landscape in the young Azorín)

\author{
José Manuel Vidal Ortuño* \\ IES Floridablanca. Murcia
}

\begin{abstract}
This article studies the landscape in regard to literary works in the young Azorín, the one from the end of the 19th century and the beginning of the 20th century, which renewed old literary clichés such as beatus ille and locus amoenus. In this new era, there is no question that, a new aesthetic (which will be expressed in two stories from Bohemia, 1897), whose postulates, undoubtedly innovative, will be put into practice in Charivari (also from 1897), through an excellent description of the Collado de Salinas, a place of refuge and flight for J. Martínez Ruiz. Such description, with variations on a theme, will be found later in other works of the writer.
\end{abstract}

Keywords: Azorín; Landscape; Beatus ille; Short stories; Bohemia; Charivari.

Resumen: Se estudia en este artículo el paisaje como materia literaria en el joven Azorín, el de finales del siglo XIX y principios del XX, renovador de viejos tópicos literarios como el beatus ille y el locus amoenus. A este tiempo nuevo le correspondería, qué duda cabe, una nueva estética (que quedará expresada en dos cuentos de Bohemia, 1897), cuyos postulados, sin duda innovadores, serán llevados a la práctica en Charivari (también de 1897), mediante una magnífica descripción del Collado de Salinas, lugar de huida y refugio para J. Martínez Ruiz. Una descripción que, como tema con variaciones, aparecerá luego en otras obras del autor.

Palabras clave: Azorín; Paisaje; Beatus ille, Cuentos; Bohemia; Charivari.

El paisajismo -acompañado o no del viejo tópico horaciano del beatus ille-fue, qué duda cabe, uno de los temas predilectos de la aún llamada generación del 98, hasta el punto de que uno de sus máximos investigadores, Pedro Laín Entralgo, nos habló en 1947 de "la invención de un paisaje" (Laín Entralgo 1998: 27). Una fascinación, la del

*Dirección para correspondencia: José Manuel Vidal Ortuño. IES “Floridablanca”. Miguel Hernández, 5. 30011 Murcia. (josemanuel.vidal@murciaeduca.es). 
paisaje, a la cual Azorín no fue ajeno y que desarrolló a lo largo de su dilatada carrera. Recordemos, a este respecto, títulos tan emblemáticos como España (1909) -que no por casualidad lleva el epígrafe de Hombres y paisajes- o la tan merecidamente alababa Castilla (1912). Sin embargo, en el presente trabajo, nos vamos a centrar en los primeros escritos de Azorín, de finales del XIX y principios del XX, cuando Azorín no era todavía Azorín, sino tan solo un joven literato que firmaba como J. Martínez Ruiz. Son estos, pues, los escritos juveniles de un escritor inconformista y rebelde, en los cuales, de tarde en tarde, asoma el gran paisajista que con el tiempo llegaría a ser.

La atracción por la naturaleza y, asimismo, por la vida retirada en el campo llamó poderosamente la atención de nuestro escritor, incluso cuando este era un niño. De sus años infantiles, Martínez Ruiz rescató la figura de don Antimo Verdú, y nos habló del mismo al menos en dos obras suyas: en Las confesiones de un pequeño filósofo (1904) y en Agenda (1959). En Las confesiones..., se ocupa de él, sin nombrarlo directamente, en el capítulo V, "El solitario", diciéndonos que este hombre abandonó Monóvar un buen día y, "amargado por las ingratitudes, se marchó al campo" (Martínez Ruiz 2005: 63-64). Medio siglo después, lo volverá a recordar en del capítulo VII de Agenda, "El casino de Monóvar", donde se mezclan, nos dice, "historia y leyenda", ya que este se retiró a una heredad y "en un altozano edificó una casita"; nuestro escritor añade a continuación que "dos hermosos alanos acompañaron siempre a don Antimo" (Azorín 1959b: 43-44). Así pues, tal vez sea don Antimo Verdú una de las más bellas, sinceras y amargas encarnaciones del viejo tópico del beatus ille.

Acaso llevado por este recuerdo -y quizás, también, por una inclinación personal-, no resulta extraño que algunos personajes azorinianos -en novelas y cuentos- decidan acabar sus días lejos del mundanal ruido; pensemos, a modo de ejemplo, en los finales de novelas como El escritor (1942) o El enfermo (1943). Personajes de sendas narraciones -Antonio Quiroga, en El escritor; Víctor Albert, en El enfermo- se retiran a casas campestres, en las que no resulta difícil adivinar lugares dilectos de la biografía de su autor, como el campo levantino o la ciudad de Petrel (Martínez del Portal 1995: 149182).

Para el primer Azorín, beatus ille y paisajismo eran algo así como las dos caras de una nueva estética; de ahí que no falte en su obra algún personaje, con vocación de artista, que haga el papel de portavoz de esta nueva sensibilidad. Vayamos, pues, a $B o-$ hemia (1897), primer libro enteramente de cuentos firmado por J. Martínez Ruiz. Como bien señaló Mirella D'Ambrosio, "el papel de la naturaleza -tan importante en la obra de Azorín- queda definido en "Paisajes", y el estudio de Castilla en su historia y en su paisaje nos lo sugiere ya en "Envidia" (Ambrosio Servodidio 1971: 15). Y así es. Porque en "Paisajes" el protagonista aspira a escribir un libro que, según sus propias palabras, hable "de toda mi vida de artista enamorado del campo, de la vegetación loca, del cielo azul, de la noche estrellada". Sus palabras, pues, se configuran como una nueva poética para los nuevos tiempos: sus estampas habrían de ser, pues, "resplandecientes de luz, con todos los ruidos de la campiña, con todos los aromas de los huertos de mi tierra". En suma: descripciones sensoriales en las que predomine la luz -y por tanto el color- y en las cuales, además, no falten tampoco notas auditivas y olfativas. Ese libro de este 
innominado artista se llamará, como no podía ser de otro modo, Paisajes; no tendrá "nada de acción ni de figuras en primer término. Paisajes solo; un álbum de acuarelas, de vistas de mi tierra" (Azorín 1959a: 313-317). Esa falta de acción, anunciada tan temprano, en sus inicios como escritor, nos lleva a pensar precisamente en novelas tan carentes de argumento del Azorín en su etapa de senectud, como La isla sin aurora y Salvadora de Olbena, ambas de 1944.

En el cuento titulado "Envidia", encontramos un motivo que será frecuente en el futuro Azorín: el paisaje visto desde la ventanilla de un tren; paisaje, por tanto, en movimiento, porque leemos que este corría e iba dejando a su paso "estaciones miserables, campesinos liados en paño pardusco". El paisaje es el de la Mancha y los ojos que observan son los de un hombre que ha sufrido un desengaño con su esposa. Por eso, los contornos del paisaje están teñidos de notas sombrías. Aparecen, por tanto, expresiones despectivas ("un mar inmenso de tierra pardusca"), una enumeración de notas negativas, unidas mediante el polisíndeton, señalando no lo que hay sino lo que falta en ese paisaje ("ni un árbol, ni una planta, ni verduras en la tierra, ni alegrías en el cielo"). Paralelamente, el narrador elige una adjetivación que guarda consonancia con su hastío vital, con su falta de esperanza: "todo triste, monótono, con la melancolía de los últimos días del otoño". A todo ello hay que añadir una coloración cenicienta, hecha a base de negros y de grises ("naturaleza sombría", "cuadro tétrico de tintes oscuros"). $\mathrm{Y}$, sin embargo, estos campos manchegos, a priori tan ásperos, tan poco amenos, son capaces de suscitar en la mente del que observa el recuerdo de la Historia, puesto que, nos dice, "hacían pensar en los amores frenéticos de un misticismo loco, en los ascetas de la Edad Media" (Azorín 1959a: 322). Unas notas llenas de poesía que nos llevan a pensar en otro fragmento de La voluntad: la descripción de la inmensa planicie yeclana del Pulpillo, tras la cual surge el recuerdo de grandes figuras históricas:

En los días grises del otoño, o en marzo, cuando el invierno finaliza, se siente en esta planada silenciosa el espíritu austero de la España clásica, de los místicos inflexibles, de los capitanes tétricos -como Alba-; de los pintores tormentarios -como Theotocópuli-; de las almas tumultuosas y desasosegadas -como Palafox, Teresa de Jesús, Larra... (Azorín 1959a: 892).

Posiblemente, el primer ejercicio de estilo de J. Martínez Ruiz, en lo que a paisaje se refiere, lo vamos a encontrar en Charivari (Crítica discordante), un folleto satírico de 1897. Si nos fijamos en su estructura, veremos que su capítulo V -tiene seis- es una especie de interludio, en el cual su protagonista -sin duda, el autor- deja momentáneamente Madrid, el de las grandes rotativas con periodistas encumbrados, donde el escritor novel pretende alcanzar la ansiada fama, y lo deja para retirarse momentáneamente a la paz del campo. Merece la pena que nos detengamos en esta descripción de Charivari, "guía y avisos del literato novel en Madrid", porque es una especie de beatus ille en medio del tráfago diario de la capital de España, con sus periódicos importantes, entre "siluetas de maestros y esbozos de jóvenes". Tal descripción fue considerada por Francisco José Martín como uno de los primeros ejercicios de estilo sobre el que se cimenta 
la fama del futuro Azorín, quien, satisfecho del resultado del mismo, decidió publicarlo, un año después, a modo de artículo titulado "Mis montañas" en Madrid Cómico (Martínez Ruiz 2000: 192-193). Y lo que leemos en ese capítulo V es la descripción de un paisaje ordenado, en virtud del buen uso de los deícticos, como si el autor -autor implícito- se encontrase dentro del mismo: "Detrás, cerrando el horizonte, una larga cadena de montañas, un inmenso telón azul, irregular, deforme, que se pierde en las nubes [...] Y delante, montes diminutos que cruzan la campiña" (Azorín 1959a: 278). Sorprende tan temprano el uso de la múltiple adjetivación, que tan característico será luego en el estilo del Azorín de principios del siglo XX.

Y si, como quedó dicho en el cuento "Paisajes", una descripción de este tipo ha de ser, ante todo, "manchas de color", vemos que estas aparecen en el texto, aunque dentro de una muy determinada gama cromática: los marrones (un monte con su "lomo pardusco"), más negros y grises ("laderas negruzcas"); mientras tanto, en claro contraste, destacan el azul de las montañas más alejadas, el "fondo rojizo" de los barrancos y, alegrando el paisaje a la manera de una ensoñación, "un lago de azul claro, casi blanco, bordeado de juncos, con álamos en las orillas". Los juncos y los álamos nos hacen presumir lo verde, que dialoga con "la verdura uniforme y brillante de los pámpanos". Lago, juncos, álamos y viñas convierten lo descrito en una especie de locus amoenus. Colores, por tanto, pero también sonidos. De hecho, sobre el silencio inmenso de esta naturaleza muda, del campo solitario y callado, surge, tenue, el sonido de la esquila de un ganado o el "ruido de abanico de una bandada de perdices" (Azorín 1959a: 279).

Mientras que para el maestro Yuste (ya en La voluntad, 1902, en el capítulo XIV de la primera parte), color y sonido no bastan, porque un paisaje, además, ha de tener "movimiento y ruido, tanto como color", veremos que en este ejercicio de estilo del primer Azorín dicho movimiento también está presente. Así, por ejemplo, en virtud de un perfecto uso de las formas verbales, las cimas de las montañas "se alejan hacia el fondo, saltando unas sobre otras, ennegreciéndose, desapareciendo, por fin, en el infinito". La descripción se aleja, además, en una especie de punto de fuga, porque "más allá de los grandes manchas de los viñedos", en el horizonte, "herido por los rayos del sol que muere, se destaca un punto brillante, como una estrella en lo oscuro de la noche: la fachada de una casita blanca". En otras ocasiones, la luz cambiante del crepúsculo, en su paulatino apagarse, le confiere al paisaje un ligero movimiento a ojos del observador: "Cae la tarde; alárgase la sombra de las montañas, que oscurecen los valles", "deslízase la claridad del púrpura del crepúsculo". Y un par de párrafos más adelante, tras la reflexión sobre el ensayista Sebastián Faure, avanza lento el anochecer, que todo lo sume en la oscuridad y hace que desaparezcan, a los lejos, "el lago azul y los grupos de árboles" (Azorín 1959a: 278-280)ํ․․

1 Desde la perspectiva que da el tiempo, calificamos este tipo de paisajes de noventayochistas. Y, sin embargo, descripciones de este tipo las podemos encontrar con anterioridad. Por ejemplo, Benito Pérez Galdós, en La familia de León Roch (1878), describe las afueras de Madrid como "vastos solares polvorientos"; es más: como un paisaje "seco, huraño, esquivo". Y, sin embargo, "esta gran estepa de Castilla" es un trasunto de "la vida espiritual surgiendo sobre la aridez del ascetismo", a la vez que "trae al pensamiento los lugares de Oriente, donde han pasado los hechos grandes de la Historia” (Pérez Galdós 1989: 833). 
Este paisaje descrito en Charivari está enclavado en un tiempo preciso, puesto que se intercala entre las anotaciones del 24 y 25 de marzo, ya que la obra adopta forma de diario. El autor nos presenta, pues, un campo en primavera, aunque no indica un espacio concreto. Solo la alusión a "mis montañas", hecha en la nota inicial de este folleto satírico, nos permite aproximarlo a la biografía del autor; este dato, más el cotejo de toda la obra de J. Martínez Ruiz. Y es que este paisaje, tan sucintamente mostrado, parece que nos remite al Collado de Salinas, finca de la familia Martínez Ruiz, próxima a Monóvar, en la zona montañosa de Alicante, y una de las constantes temáticas a lo largo de toda la obra de Azorín. Llegamos a esa conclusión al cotejar estas páginas primeras -aunque en absoluto primerizas- con otras cercanas en el tiempo, como las que se hallan en Diario de un enfermo, novela corta de 1901, y Antonio Azorín, novela de 1903.

En Diario de un enfermo, Martínez Ruiz toma como base la descripción paisajista que hemos visto en Charivari, dándole otra vuelta de tuerca, con el ánimo de perfeccionarla. No suma más que tres entradas en esta nouvelle escrita en forma diarística: las del 20 de julio, 20 de agosto y 2 de septiembre; tres días distintos y en horas del día también distintas (un anochecer, una amanecer y una terrible tormenta que acaece en medio de la noche). Sin embargo, lo que nos llama la atención ahora es que el escritor intenta ubicar lo descrito en medio de una geografía más concreta, en "plena montaña levantina", en "inmenso collado", donde los agricultores que en ella trabajan se expresan en una lengua que no es el castellano, sino el valenciano ("Palmeres per baix, señal d'aigüa"). La casita blanca que en la descripción de Charivari hacía como una especie de punto de fuga en el horizonte, ha sido sustituida en esta otra por "el triángulo rojizo de un castillo moruno", el cual "luce a los postreros rayos de sol como un grano de oro" (Azorín 1959a: 707).

Con todo, será en Antonio Azorín (1903) donde la toponimia auténtica le gane terreno a lo indeterminado, situando esta descripción, que ahora abre la novela, en una realidad más real y, por ende, más querida. Así pues, ahora se nos habla de las Lometas, del cabezo árido de Cabreras, del monte de Castalla, mientras que, "en el centro, sobre el azul del fondo, resalta el ingente peñón de Sax, coronado de torreón moruno". Con el paso de las horas, "el dorado castillo refulge en su postrer destello y desaparece". La sierra de Cabreras, el monte de Castalla, la fortaleza de Sax vista al fondo del valle sitúan con total seguridad este fragmento, heredero de los otros dos anteriores, en el Collado de Salinas, lugar ameno y agradable, donde nuestro escritor pasó horas y horas durante su niñez y allá en su mocedad, que, con el correr de los años, acabaría convirtiéndose en añorado y anhelado beatus ille donde poder huir de las tribulaciones de este mundo. Un anhelo que nuestro escritor, ya anciano, no pudo conseguir, pero que sí lograron algunos de sus personajes de ficción, como el protagonista del cuento "El extranjero en su patria" (Pensando en España, 1940) o Antonio Quiroga (personaje de la novela El escritor, que antes he mencionado).

En definitiva, a finales del siglo XIX y a principios del XX, los nuevos escritores pensaron en el paisaje como elemento importante de la nueva estética, "una emoción -pondrá Azorín en boca del maestro Yuste, una vez más- completamente, casi comple- 
tamente moderna". He querido mostrar en este trabajo cómo el joven Martínez Ruiz pergeñó una especie de poética en un cuento, "Paisajes", y describió las llanuras de la Mancha como estado del alma en otro relato, "Envidia" (ambos en Bohemia, de 1897). Como autor que se deja parte de sus vivencias en sus escritos, describió un lugar harto conocido y muy amado, el del Collado de Salinas, ofreciéndolo a sus lectores, a modo de un tema con variaciones, primero en Charivari, luego en Diario en un enfermo, y por último en Antonio Azorín.

\section{BIBLIOGRAFÍA}

AMBROSIO SERVODIDIO, Mirella de (1971): Azorín, escritor de cuentos. Nueva York: Las Américas.

AZORÍN (1959a): Obras completas, vol. I. Madrid: Aguilar.

(1959b): Agenda. Madrid: Biblioteca Nueva.

LAÍN ENTRALGO, Pedro (1998): La generación del 98. Madrid: Espasa Calpe.

MARTÍNEZ DEL PORTAL, María (1995): "Sobre un aspecto de las novelas de Azorín: los desenlaces". Anales Azorinianos, 4: 149-182.

MARTÍNEZ RUIZ, José (1997): La voluntad. Ed. de María Martínez del Portal, Madrid: Cátedra.

(2000): Diario de un enfermo. Ed. de Francisco José Martín, Madrid: Biblioteca Nueva.

(2005): Las confesiones de un pequeño filósofo. Ed. de María Martínez del Portal, Madrid: Biblioteca Nueva.

PÉREZ GALDÓS, Benito (1989): Obras completas, vol. I. Madrid: Aguilar.

\section{PERFIL ACADÉMICO Y PROFESIONAL}

José Manuel Vidal Ortuño es doctor por la Universidad de Murcia, título que alcanzó con una tesis sobre la cuentística de Azorín, dirigida por la profesora Ana L. Baquero Escudero, catedrática de Literatura Española. Fruto de esa labor son ediciones críticas de dos obras de J. Martínez Ruiz, como El buen Sancho (Biblioteca Nueva, 2004) y España (Biblioteca Nueva, 2010), más un ensayo titulado Los cuentos de José Martínez Ruiz (Azorín), publicado por la Universidad de Murcia, en el 2007, iniciándose con él la colección Signos. Sus trabajos se centran, principalmente, en la literatura española contemporánea. Algunos de sus trabajos, que giran en torno a los más diversos escritores (el ya nombrado Azorín, Luis Cernuda, Gerardo Diego, Elena Quiroga...), fueron apareciendo, desde hace años, en revistas como Montearabí, Monteagudo, Rilce, Murgetana o Razón y Fe, entre otras. Desde 1989 es Profesor de Enseñanza Secundaria y en la actualidad da clases del Lengua y Literatura Españolas en el Instituto "Floridablanca", de Murcia.

Fecha de recepción: 07/05/2020

Fecha de aceptación: 23/06/2020 